\title{
Temperaturas de armazenamento de alimentos em estabelecimentos comerciais na cidade de Santa Maria, RS*
}

\author{
Temperature of food storage in commercial stores in Santa Maria, RS, Brazil \\ Lisandra Mürmann ${ }^{1}$, Carlos Augusto Mallmann² \& Paulo Dilkin²
}

\begin{abstract}
RESUMO
A temperatura correta do armazenamento de alimentos é de extrema importância para a sua conservação, sendo este um dos itens essenciais na prevenção das toxinfecções alimentares. O presente estudo teve como objetivo avaliar as temperaturas dos equipamentos de conservação nos estabelecimentos que comercializam alimentos na cidade de Santa Maria, RS. A avaliação consistiu em um estudo descritivo observacional, onde se inspecionou cada unidade de observação durante o período de abril a outubro de 2003. Foram verificadas as temperaturas de 506 equipamentos de armazenamento de alimentos, sendo 327 de resfriamento, 173 de congelamento e 6 estufas de aquecimento pertencentes a 154 estabelecimentos comerciais de Santa Maria, RS. As aferições foram realizadas com termômetro à laser e termômetro digital. Também foi utilizado um termohigrômetro para medir a temperatura interna do alimento, temperatura ambiente e umidade. Os equipamentos de resfriamento apresentaram temperaturas médias inadequadas em $29,7 \%$ dos estabelecimentos, sendo a câmara fria o equipamento que apresentou o maior índice de adequação $(94,2 \%)$. Os equipamentos de congelamento dos alimentos apresentaram alto índice de temperatura inadequada $(86,1 \%)$ e todas as estufas de aquecimento analisadas apresentaram-se inadequadas. Não houve correlação entre a temperatura do ambiente e umidade com a temperatura do equipamento, bem como entre a temperatura interna do alimento com a do equipamento. Este estudo mostrou que os conservadores de alimentos nos estabelecimentos comerciais de Santa Maria, RS apresentaram alto índice de inadequação em suas temperaturas, um problema que pode causar sérios riscos à saúde do consumidor, necessitando de uma vigilância mais eficiente.
\end{abstract}

Descritores: temperatura, alimentos, Saúde Pública, armazenamento.

\section{ABSTRACT}

The correct temperature of storage is very important for food conservation, which is essential for the prevention of foodborne diseases. The present study aimed to evaluate the range in temperature in storage equipment used in grocery and food stores in Santa Maria, RS/Brazil. The evaluation was an observation-descriptive study during the period from April to October 2003. The temperature of 506 pieces of equipment was verified, including 327 refrigerating units, 173 freezing units, and 6 stoves in a total of 154 establishments in Santa Maria. Measurements of the internal temperature of the foodstuff, the room temperature and humidity were taken using a laser thermometer, a digital thermometer, and a thermo-hygrometer, respectively. The refrigerating units presented inadequate mean temperatures in $29.7 \%$ of the establishments, with the cold chamber as the most reliable piece of equipment, with a high reliability index (94.2\%). The freezing units presented high disagreement index (86.1\%) and all of the hot stoves presented inadequate temperatures. No correlations were detected between room temperature and humidity, and the temperature of the pieces of equipment, and between the internal temperatures of the foodstuff and the temperatures of the sets of equipment. This study showed that the conservation of food in stores in Santa Maria presented an elevated index of inadequate temperature, a problem that could cause serious risks to the consumer's health, highlighting the need for a more efficient surveillance.

Key words: temperature, foods, Public Health, storage. 


\section{INTRODUÇÃO}

Todos alimentos, de origem animal ou vegetal, apresentam os mais diversos tipos de microorganismos, os quais fazem parte de sua microbiota habitual. Para manter o processo de multiplicação, esses microorganismos necessitam de condições favoráveis, representadas por múltiplos fatores, entre eles a temperatura [4], que durante o processo de conservação é de fundamental importância para inibir a proliferação microbiana e minimizar as reações químicas que causam a deterioração dos alimentos [3,4,15]. Os valores corretos de temperatura de armazenamento, conforme o Decreto $\mathrm{n}^{\circ} 23.430$, são de $7^{\circ} \mathrm{C},-18^{\circ} \mathrm{C}$ e $60^{\circ} \mathrm{C}$ para refrigeração, congelamento e estufas, respectivamente [10].

As Toxinfecções Alimentares (TIA's) podem ser resultantes da conservação e manipulação incorretas. A etapa onde ocorre a contaminação dos alimentos, ou seja, a qual o agente contaminante sobrevive ou se multiplica é dependente de uma série de variáveis. A identificação da causa que levou o microorganismo a sobreviver e se multiplicar no alimento, é de fundamental importância para a adoção de medidas preventivas no que se refere ao comércio de alimentos [14].

Este estudo objetivou avaliar as temperaturas dos equipamentos de conservação de alimentos de estabelecimentos da cidade de Santa Maria, RS, tentando identificar possíveis riscos para a segurança alimentar dos consumidores.

\section{MATERIAIS E MÉTODOS}

As inspeções foram realizadas em 154 estabelecimentos que comercializavam alimentos na cidade de Santa Maria-RS, no período de abril a outubro de 2003, sendo 29 mercados, 54 armazéns, 12 açougues, 13 padarias, 22 lancherias e 24 restaurantes. As temperaturas foram avaliadas conforme Legislação Sanitária, Lei Estadual no 6.503, Decreto $n^{\circ} 23.430$ [10]. Todos os equipamentos de armazenamento, tais como: refrigeradores (145), câmaras frias (52), balcões refrigerados (145), congeladores (136), balcões de congelamento (30) e estufas (6) encontrados nos estabelecimentos foram verificados, medindo-se a temperatura interna de cada um.

A temperatura de cada equipamento foi obtida através da média de 10 aferições realizadas em diversos pontos aleatórios de seu interior. O termômetro utilizado foi o laser Minitemp. Em alguns alimentos, como a carne, onde foi introduzido um termômetro de profundidade (tipo espeto), a tempera- tura interna do alimento também foi verificada; para este procedimento utilizou-se um termômetro Minipa MV-363.

A temperatura ambiente e umidade relativa do ar foram medidas no local onde o equipamento se encontrava, com um termo-higrômetro Minipa Mt-241.

As avaliações estatísticas obtidas foram realizadas empregando-se a estatística descritiva (média, desvio padrão, máxima, Qui-quadrado e correlação), com o auxílio do programa estatístico Statgraphics, versão 5.0, 2000. Dos pontos médios de temperatura foi utilizado o método estatístico Qui-quadrado $(\mathrm{P}<0,05)$, para o número de equipamentos com a temperatura em acordo ou desacordo e entre os diferentes tipos de estabelecimentos.

Entre a temperatura ambiente/umidade e temperatura dos equipamentos, bem como temperatura interna do alimento e temperatura dos equipamentos, estipulou-se adicionalmente o coeficiente de correlação $\left(r^{2}\right)$.

\section{RESULTADOS}

Observou-se que $24,8 \%$ dos equipamentos de resfriamento apresentaram temperaturas médias inadequadas, segundo o recomendado pela Legislação Sanitária Estadual. Os resultados indicam diferença entre os tipos de equipamentos, sendo a câmara fria o equipamento que apresentou maior índice de adequação $(94,2 \%)$. Os equipamentos responsáveis pelo congelamento dos alimentos (balcões de congelamento, congeladores e câmaras de congelamento) apresentaram na sua grande maioria $(92,4 \%)$, temperaturas inadequadas para a conservação de alimentos. As estufas de conservação de alimentos aquecidos estavam todas em temperatura inadequada, tendo sido encontrado como máximo a temperatura de $41,9^{\circ} \mathrm{C}$ (Figura 1).

A média de temperatura verificada nos equipamentos de refrigeração encontrou-se adequada, mas os equipamentos de congelamento apresentaram médias acima do permitido pela legislação (Tabela 1). As médias bastante altas, demonstradas nesta tabela, permitem inferir que muitos equipamentos poderiam estar desligados ou funcionando inadequadamente.

Como se constata na Figura 2, quase metade dos equipamentos de conservação analisados nos estabelecimentos da cidade de Santa Maria (47,5\%) está operando com temperatura em desacordo com a Legislação vigente. Os açougues foram os estabelecimen- 
Tabela 1. Temperaturas médias e temperaturas máximas encontradas em diferentes tipos de equipamentos nos estabelecimentos da cidade de Santa Maria, RS, no período de abril a outubro de 2003 .

\begin{tabular}{lcc}
\hline Tipos de Equipamentos & $\begin{array}{c}\text { Temperatura } \\
\text { média }\left({ }^{\circ} \mathrm{C}\right)\end{array}$ & $\begin{array}{c}\text { Temperatura } \\
\text { máxima }\left({ }^{\circ} \mathrm{C}\right)\end{array}$ \\
\hline Câmara Fria & $1,11(3,63)^{*}$ & 15,25 \\
Balcão refrigerado & $4,62(3,86)$ & 13,60 \\
Refrigerador & $5,21(1,93)$ & 16,15 \\
Balcão de congelamento & $-8,58(6,26)$ & 5,90 \\
Congelador & $-11,67(6,46)$ & 1,30 \\
Câmara de congelamento & $-9,57(3,51)$ & $-5,35$ \\
Estufa & $30,65(10,91)$ & 41,90 \\
\hline "Desvio Padrão & & \\
\end{tabular}

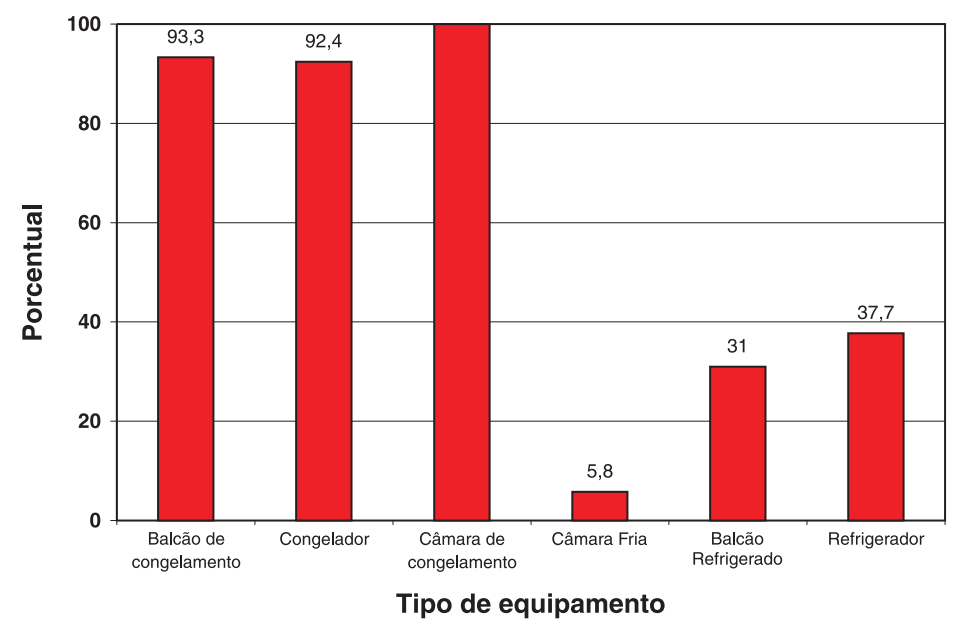

Figura 1. Porcentual de equipamentos que apresentavam temperatura irregular, investigado em estabelecimentos comerciais de Santa Maria, RS, no período de abril a outubro de 2003.

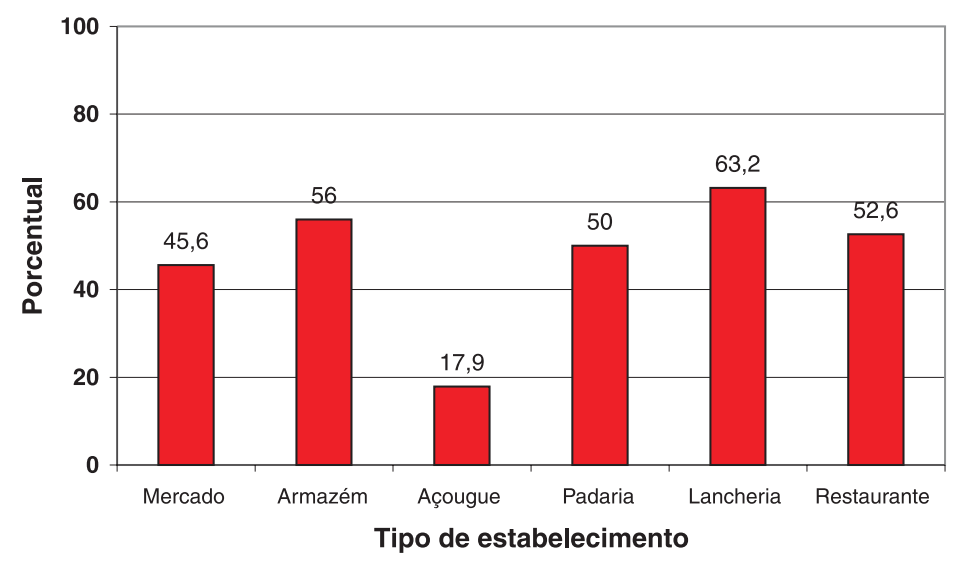

Figura 2. Porcentual de equipamentos com temperatura irregular encontrados, conforme o tipo de estabelecimento na cidade de Santa Maria, RS, no período de abril a outubro de 2003. tos que apresentaram o maior índice $(82,1 \%)$ de equipamentos em temperatura adequada entre todas as categorias de estabelecimentos inspecionadas.

Foi também realizada a medição da temperatura ambiente e da umidade do local em que cada equipamento se encontrava, sendo que não observou-se um coeficiente de correlação significativo $\left(\mathrm{r}^{2}\right)$. A temperatura interna de alguns alimentos também foi avaliada, mas não se obteve coeficiente de correlação significativo $\left(r^{2}=0,6128\right)$ em relação à temperatura do equipamento.

\section{DISCUSSÃO}

$\mathrm{O}$ armazenamento de alimentos em temperatura adequada é imprescindível para mantêlos por períodos prolongados. Os alimentos podem ser inspecionados de acordo com as técnicas mais modernas, mas se não forem armazenados adequadamente no varejo podem perder toda a sua qualidade em razão da estocagem [4].

O uso correto dos equipamentos de frio reduz significativamente a deterioração dos alimentos e os riscos à saúde do consumidor [5]. Quanto menor a temperatura de armazenamento, mais lentas serão as reações químicas, as atividades enzimáticas, a multiplicação dos microorganismos e maior será o tempo que os alimentos poderão ser armazenados $[11,13]$.

Em vistorias realizadas a 39 estabelecimentos varejistas de São Paulo/SP, foram encontradas altas taxas de inadequação, tanto nos equipamentos de refrigeração $(60,6 \%)$, como nos equipamentos de congelamento $(89,1 \%)$ [8]. Um dos maiores problemas encontrados nas condições de conservação pelo frio em estabelecimentos que comercializam perecíveis foi o mau funcionamento dos equipamentos, que ocorre tanto em redes de grandes supermercados como em pequenos estabelecimentos de bairros populares e periféricos das grandes capitais [7].

$\mathrm{O}$ período em que os alimentos podem ficar refrigerados depende da sua carga microbiana antes da estocagem, do grau de perecibilidade e o tipo de armazenagem. Alimentos frescos crus, recentemente cozidos com baixas contagens microbianas podem ser estocados por três a quatro dias, antes dos microorganismos 
causarem sua deterioração [4]. No entanto, alimentos como verduras, algumas frutas e carne fresca, supostamente em razoável condição de higiene, podem ficar armazenados até sete dias na temperatura correta [9]. Conforme dados destes autores, constata-se que os alimentos comercializados em Santa Maria podem estar tendo um tempo de conservação reduzido, ou estão sendo comercializados em processo de deterioração, pois não estão sendo armazenados em temperatura correta, o que pode acarretar perdas aos proprietários e danos à saúde do consumidor.

$\mathrm{O}$ congelamento tem como finalidade prolongar a conservação do alimento, em relação àquelas conseguidas apenas com a refrigeração. As temperaturas utilizadas são baixas o suficiente para reduzir ou cessar a deterioração causada pelos microorganismos, enzimas ou agentes químicos como o $\mathrm{O}_{2}$. $\mathrm{O}$ congelamento é um dos melhores métodos para manter a cor, aroma e aparência de muitos alimentos [5]. Os altos índices de inadequação nas temperaturas de congelamento encontradas neste trabalho são alarmantes, podendo interferir na qualidade dos produtos comercializados, possibilitando TIA's e perdas das características nutricionais e organolépticas dos alimentos.

Em pesquisa realizada em Niterói/RJ foram avaliadas as temperaturas dos equipamentos de armazenamento em 20 mercados, encontrando uma baixa correlação $\left(r^{2}=0,2969\right)$ entre a temperatura das gôndolas e dos queijos [12]. Da mesma forma, no presente estudo, observou-se uma baixa correlação da temperatura interna do alimento com a temperatura do equipamento. Um dos prováveis motivos para estes resultados ter sido a recente colocação de muitos alimentos nas gôndolas, não havendo tempo suficiente para seu resfriamento interno ou até mesmo de sua superfície.

Muitos alimentos são mantidos quentes em estufas de ar quente até o momento de serem comercializados. Todavia, quando o equipamento de aquecimento é mal conservado ou utilizado de modo inadequado, o intervalo de tempo entre o preparo e o consumo favorece a incubação de eventuais agentes microbianos patogênicos, possibilitando a sua multiplicação ou a produção de toxinas [4]. Observou-se, neste estudo, que a totalidade das estufas analisadas (6) apresentavam-se em temperaturas inadequadas.

Conforme estudo realizado em Fortaleza/CE, observou-se que 52,5\% das estufas analisadas em 21 lanchonetes estavam com a temperatura inadequada [6]. Verificou-se, no Estado do Rio Grande do Sul, que as temperaturas das estufas em estabelecimentos que comercializam alimentos apresentavam em $89,4 \%$ dos equipamentos analisados valores irregulares, sendo que destes, $26,3 \%$ apresentavam temperatura entre $30^{\circ} \mathrm{C} \mathrm{e} 40^{\circ} \mathrm{C}$, faixa considerada ótima para o desenvolvimento de bactérias mesófilas [1]. Foram encontrados índices de inadequação $(91,7 \%)$ similares ao deste trabalho nas estufas dos estabelecimentos na cidade de Uberaba/MG [2].

\section{CONCLUSÃO}

Conclui-se que a maioria dos equipamentos de conservação dos alimentos dos estabelecimentos comerciais de Santa Maria-RS, está operando em temperatura inadequada, constituindo um problema que pode causar riscos à saúde do consumidor, sendo necessária a adoção de medidas da Vigilância Sanitária para correção deste problema.

\section{REFERÊNCIAS}

1 Almeida F.S. \& Lagaggio V.R.A. 2001. Avaliação das temperaturas de estufas para conservação de alimentos em estabelecimentos no município de Santa Maria, RS. Higiene Alimentar. 15: 98.

2 Chesca A.C. 2000. Avaliação da temperatura das estufas de salgados de bares e lanchonetes do Município de Uberaba, MG. Higiene Alimentar. 14: 87-89.

3 Ferrari C.K.B. \& Torres E.A.F.S. 2000. Parâmetros de qualidade em produtos de origem animal comercializado no município de São Paulo. Higiene Alimentar. 14: 25-32.

4 Germano P.M.L. \& Germano M.I.S. 2001. Higiene e Vigilância Sanitária de Alimentos. São Paulo: Varela, 629p.

5 Hobbs B.C. \& Roberts D. 1999. Toxinfecções e controle higiênico-sanitário de alimentos. 4.ed. São Paulo: Varela, 376p.

6 Lima G.B. \& Chagas C.M.A. 2003. Pontos críticos em preparações comercializadas em lanchonetes dos campus de duas universidades da cidade de Fortaleza, CE. Higiene Alimentar. 17: 97.

7 Mendes A.C.R., Santana L.G., Costa D.S. \& Almeida J.F. 2001. Condições de comercialização de cortes cárneos em supermercados da cidade de Salvador-BA. Aspectos higiênico-sanitários e de conservação. Higiene Alimentar. 15: 58-62.

8 Pavanelli L.C., Villela V.H., Souza S.S., Clemente R.M., Leal R.M. \& Azevedo J.S. 2003. Diagnóstico das condições de equipamentos de frio em estabelecimentos comerciais varejistas do município de São Paulo. Higiene Alimentar. 17: 148. 
9 Riedel G. 1996. Controle sanitário dos alimentos. 2.ed. São Paulo: Atheneu, 320p.

10 Rio Grande do Sul. Decreto n'23.430 de 24 de outubro de 1974. Regulamento sobre a promoção, proteção e recuperação da saúde pública. Diário Oficial do Estado do Rio Grande do Sul]. Publicado em 03/12/74.

11 Silva Junior E.A. 1997. Manual de controle higiênico-sanitário em alimentos. 2.ed. São Paulo: Varela, 385p.

12 Tavares D.S.B, Ribeiro S.F., Silva T.P. \& Abreu L.R. 2003. Avaliação da temperatura de conservação de queijos tipo Minas Frescal no mercado varejista do município de Niterói-RJ. Higiene Alimentar. 17: 209.

13 Thomson J.E., Whitehead W.K. \& Mercuri A.J. 1974. Chilling poultry meat - a literature review. Poultry Science. 53: 12681281.

14 Todd E.C., Guzewich J.J. \& Bryan F.L. 1997. Surveillance of foodborne disease IV. Dissemination and uses of surveillance data. Journal of Food Protection. 60: 715-723.

15 Toledo R.T. 1999. Fundamentals of food process engineering. 2nd.edn. Aspen: Maryland, 602p. 